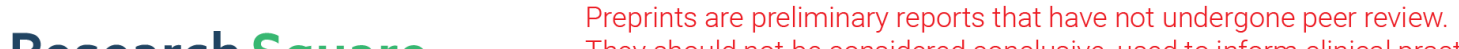 They should not be considered conclusive, used to inform clinical practice, or referenced by the media as validated information. \\ Clustering and Graph Mining Techniques for Classification of Complex Structural Variations in Cancer Genomes
}

Gonzalo Gómez-Sánchez ( $\sim$ gonzalo.gomez@bsc.es )

Barcelona Supercomputing Center https://orcid.org/0000-0002-0985-1632

\section{Luisa Delgado-Serrano}

Barcelona Supercomputing Center: Centro Nacional de Supercomputacion

\section{David Carrera}

Barcelona Supercomputing Center: Centro Nacional de Supercomputacion

\section{David Torrents}

Barcelona Supercomputing Center: Centro Nacional de Supercomputacion

Josep Lluis Berral

Barcelona Supercomputing Center: Centro Nacional de Supercomputacion

\section{Research}

Keywords: complex rearrangements, structural variants, cancer genomics, clustering, graph mining, motif finding

Posted Date: May 5th, 2021

DOl: https://doi.org/10.21203/rs.3.rs-476852/v1

License: (9) (1) This work is licensed under a Creative Commons Attribution 4.0 International License. Read Full License 


\title{
Clustering and Graph Mining Techniques for Classification of Complex Structural Variations in Cancer Genomes
}

\author{
Gonzalo Gomez-Sanchez ${ }^{1,2^{*} \dagger}$, Luisa Delgado-Serrano ${ }^{3 \dagger}$, David Carrera ${ }^{1}$, David Torrents ${ }^{3,4}$ and Josep LI. \\ Berral ${ }^{1,2 *}$
}

\footnotetext{
${ }^{*}$ Correspondence: gonzalo.gomez@bsc.es; josep.berral@bsc.es

${ }^{1}$ Barcelona Supercomputing Center (BSC), Department of Computer Science, Barcelona, Spain

† Gonzalo Gomez-Sanchez and Luisa Delgado-Serrano contributed equally to this work.
}

\begin{abstract}
Background: For many years, a major question in the field of cancer genomics has been the identification of those variations that can have a functional role in cancer, and distinguish from the majority of genomic changes that have no functional consequences. This is particularly challenging when considering complex chromosomal rearrangements, which are often composed of multiple DNA breaks, resulting in difficulties to classify and interpret them functionally. Despite recent efforts towards the classification of structural variants (SVs), more robust statistical frames are needed to better classify these variants, and to isolate those that derive from specific molecular mechanisms.

Results: We present a new statistical approach to analyze SVs patterns from 2392 real tumor samples from the Pan-Cancer Analysis of Whole Genomes (PCAWG) Consortium and to identify significant recurrence, which can inform of relevant mechanisms involved in the biology of tumors. The method is based on recursive KDE clustering of $152,926 \mathrm{SV}$, graph mining techniques and statistical measures. The proposed methodology was able not only to identify complex patterns but also to prove them as not random occurrences. Furthermore, a new class of pattern that was not previously described has been identified.

Conclusions: We developed a new and unbiased methodology for clustering SVs to search further for complex patterns by using a cost-efficient graph mining method. Followed by deep statistical analysis and applying randomization techniques, our proposed framework allows for discerning between stochastic chromosomal rearrangements and complex patterns that might have specific underlying mechanisms present in different cancer types.
\end{abstract}

Keywords: complex rearrangements; structural variants; cancer genomics; clustering; graph mining; motif finding

\section{Background}

Cancer is a disease mainly driven by genetic alterations that take place in somatic cells. The somatic mutations in a cancer cell genome involve several distinct classes of DNA sequence change. A particularly important class of somatic mutations are structural variations that consist of genomic rearrangements, such as large deletions, large insertions, tandem duplications, inversions, and translocations [1]. In the last decade, whole-genome analysis has shown that several SVs are not independent events driven by genome instability, but they are acquired through a "single-hit" event involving several DNA breaks, usually resulting in complex genome rear- 
rangements. However, there is not a standard methodology to identify recurrent and statistically significant patterns of SVs, being the key for understanding the underlying mechanisms of complex rearrangements, by which they might contribute to the development of tumors.

A few classes of complex chromosomal rearrangements have been described in tumor genomes. For example, in 2011, Stephens and co-workers described an SV pattern characterized by multiple (sometimes hundreds) rearrangements that occur within a restricted portion of the genome, involving normally one, but also rarely two chromosomes [2]. In another study, Baca and co-workers reported another specific pattern of chromosomal rearrangements in prostate tumors called Chromoplexy, which is characterized by a closed chain of translocations involving several chromosomes [3]. Recently, the PCAWG Consortium collected whole genome sequencing data from 2392 cancers across 36 tumor types, produced by the International Cancer Genome Consortium (ICGC) and The Cancer Genome Atlas (TCGA) projects [4]. There, Li and co-workers described a replication-based mechanism of structural variation that results in varied chromosomal structures with low-level copy number gains and recurring inverted rearrangements [5]. Despite all these efforts to classify and characterize these complex events, a major fraction of the identified SVs in the PCAWG study remained "unclassified."

In order to fulfill this gap, we developed an innovative statistical approach to be able to discriminate between stochastic chromosomal rearrangements, probably due to general genome instability, from those patterns that might have specific and recurrent molecular mechanisms behind. The generation of such a workflow will allow the overall improvement of classification methods for the discrimination of mutations and to identify particular SV signatures as markers of tumor formation and progression.

Here, we applied this new statistical frame to 2392 tumor genomes from the PCAWG Consortium, including more than 152,926 SVs. This method takes into account the local distribution of SVs in every sample and is optimized using the global distribution across the dataset, using a Kernel Density Estimation function $[6,7]$. The aim of the clustering is to join the rearrangements that are likely derived from the same molecular mechanism, as they share some topological properties. We assessed that the clustering approach joins rearrangements not randomly by performing a permutation test. Then, we provided a graph mining method to analyze the SV patterns, using advanced high performing technologies to reduce the computational cost $[8,9]$. Finally, we adapted a methodology proposed by Wong [10] to obtain the level of significance of the different patterns based on the Abundance, a measure that indicates the overrepresentation or underrepresentation of a pattern against a random scenario.

By overcoming currently unsolved challenges of SVs classification in cancer, our results provide insights into different complex patterns of SVs emerging from possible chromatin conformations that allow interactions of different genomic regions that are occurring in a cancer cell.

\section{Methods}

Our main strategy for the identification of complex chromosomal rearrangements is summarized in Fig. 1. Preceded by a quality check and pre-processing of the 
PCAWG data, the main workflow is composed of three major steps: KDE clustering, graph mining, and motif finding.

Defining clusters to identify the SVs involved in complex rearrangements The clustering method was based on Kernel Density Estimation (KDE) [6, 7], a nonparametric statistical method to estimate the probability density function of a random variable. In this study, the random variable is the position of the SV, which is defined by the points where the rearrangements occur in the genome (breakpoints). Clustering those breakpoints that correspond to the same single rearrangement event is crucial to later classify complex patterns of SVs. We chose this clustering method because it uses a density estimation of the breakpoints as a starting point, which allowed us to rely both on the closeness of the breakpoints and their density. Using the Gaussian Kernel based on normal distribution, the only hyperparameter to be set was the bandwidth $[11,12]$. This value defines how the density estimation is going to be: increasing the bandwidth leads to bigger clusters, whereas low values generate smaller and sparser clusters. The final size of each cluster will depend on both the selected bandwidth and the density of the breakpoints for each particular case. We had to set a bandwidth that provides the lowest intra-cluster distance, defined as the highest distance between two breakpoints within the same cluster, and the highest inter-cluster distance, defined as the lowest distance between two breakpoints of adjacent clusters, both illustrated in Fig. 2. Optimizing these two distances makes the clusters to be as separate from each other as possible and as small as possible. These distances were obtained for all samples at the same time, fixing the same bandwidth value for every sample. Therefore, taking into account the global breakpoints distribution across all the samples to set the bandwidth value, we were able to avoid potential biases derived from a particular sample distribution and to join together two clusters or not when needed.

Since the human genome is organized into 23 pairs of chromosomes, we performed the clustering locally at every chromosome. Figure 2 shows how the method works using different bandwidth values on the same region of a given chromosome. Once the clustering was done, the next step was to locate all the peaks of the function and assign the breakpoints to the closest peak. These peaks represent the cluster centers to use for all the breakpoints assigned to each cluster at the graph mining step (see below).

In order to improve the clustering resolution, a recursive 2-step clustering was carried out: after the first KDE clustering process, we performed a second clustering inside every cluster. To avoid already described complex patterns, such as Chromothripsis, the breakpoints looping over the same region were discarded after the first clustering round. This process made the mining of motifs computationally more efficient, avoiding noise into the second step of the clustering. In the second round of clustering, different bandwidth hyper-parameters were set to compare intra-cluster and inter-cluster distances. Since the clustering method was based on a density estimation function, we ensured a linear growth of the number of operations with the increase of data. Since both the density estimation and the final cluster selection only interact with data from a region of the chromosome at a time, the number of operations of the method will always be smaller than $n^{2}$, where $n$ is the 
number of breakpoints, avoiding high computational expenses. To provide a better understanding of the method, the pseudocode of the full clustering process can be found in Algorithm 1.

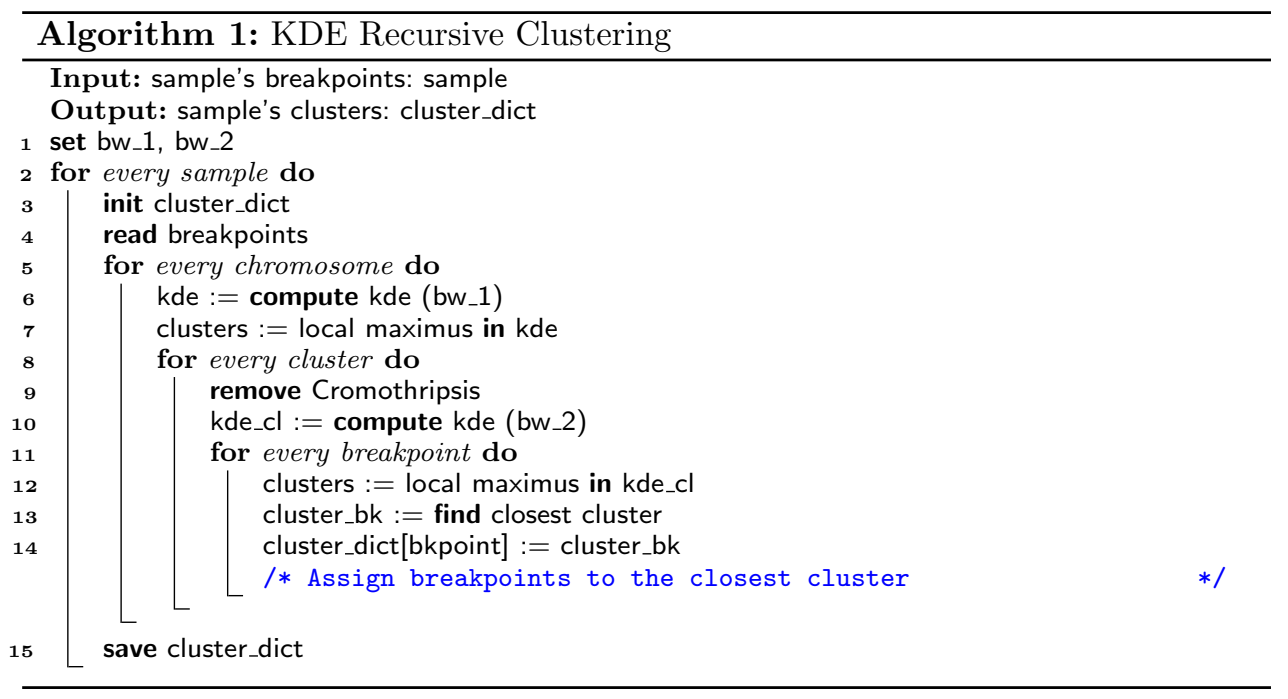

We validated that our clustering approach was not joining random SVs by performing two tests. First, we generated simulated datasets 100 times by pooling together all the breakpoints of the samples, and creating new samples with random rearrangements. In the first test, we estimated the average dispersion of breakpoints in each simulated dataset. We used as a dispersion measure the standard deviation of the difference of base pair between adjacent breakpoints in a chromosome. Then, we compared the average dispersion distribution from the simulated datasets against the average dispersion of breakpoints in the original dataset performing a one sample Z-Test. In the second test, we applied the KDE clustering method to each simulated dataset as described for the original dataset. For each permutation we calculated the average cluster density defined as the average number of breakpoints per cluster and compared to the average number of breakpoints per cluster in the original dataset using a one sample Z-Test.

\section{Graph Mining to search for complex rearrangements}

The clustering process set out every sample as a graph where the breakpoint clusters are represented as vertices and the edges connecting these vertices correspond to the rearrangements. Since vertices could be composed of several breakpoints from different rearrangements, different graphs could be generated. To narrow down the survey of graphs, we focused only on Hamiltonian cycles (mentioned further only as cycles), where every vertex is connected to two other vertices (Fig. 3).

To find and count rearrangement patterns inside each graph, we used a search approach method based on the VSIGRAM method [13], following a vertical approach and finding the frequent subgraphs in a depth-first fashion. As the subgraph mining problem becomes computationally hard (NP-hard), we performed a pruned search with $\max$ size $=6$. The graph-based data mining for SV pattern searching includes four steps: deduplicate edges, generate the graph, subgraph mining, and reduce similar patterns. 
Deduplicate edges

Since every cluster can include more than one breakpoint, it is likely to find clusters with more than one edge going to one another cluster. These edges were therefore duplicated and had to be deduplicated, simply removing all of them except one.

Generate the graph

Next, we generated graphs for each sample, considering the cluster centers as the vertices, and the unique edges as the connecting edges of the vertices.

\section{Subgraph mining}

The used method for subgraph mining visited the graph through depth-first search, allowing parallelism, e.g. by splitting each starting vertex to be processed at the same time. At every vertex, we looked for all the possible connected paths of size 1 . Then, these subgraphs were the candidates for looking for all the possible connected paths of size 2 . The process was repeated for the paths of sizes $3,4,5$, and 6 . A graphic representation of this process can be found in Fig. 4 and the corresponding pseudocode in Algorithm 2.

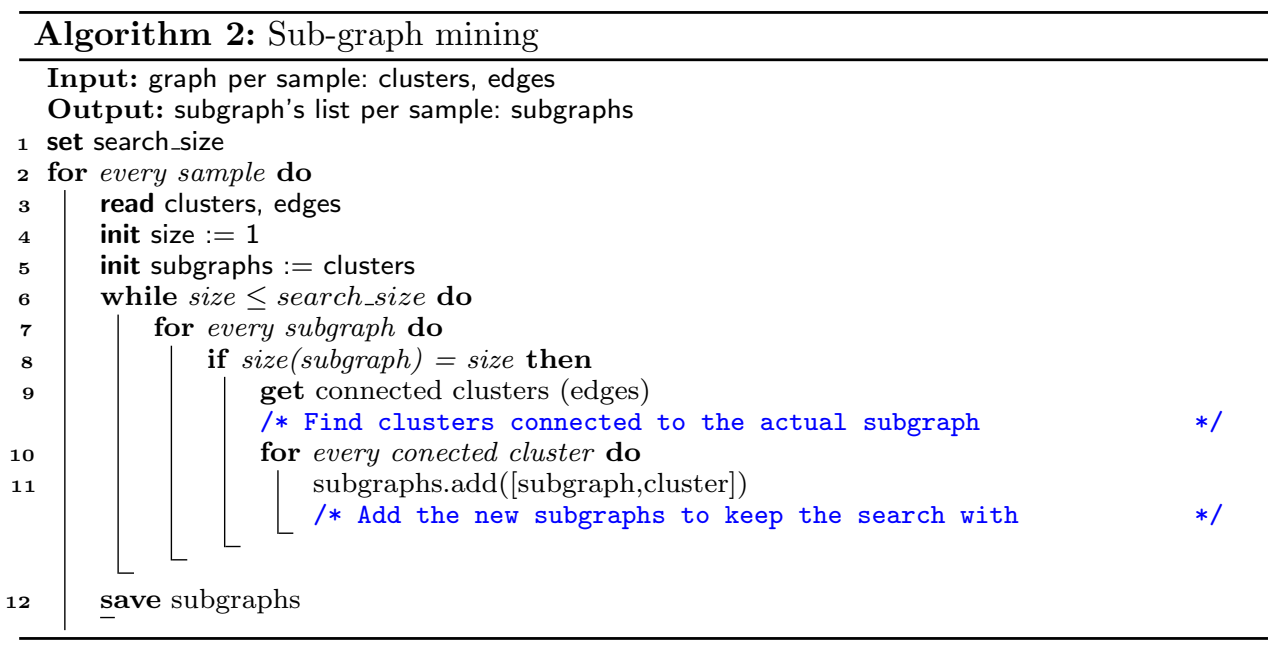

Reduce similar patterns

All of the subgraphs obtained from the vertices from a given sample were stored together and duplicated cases were eliminated by matching canonical labels and edge hashes.

\section{Defining statistically significant patterns}

In order to discern statistically significant patterns from random distributions, we compared frequencies between real observations and random observations from simulated datasets using a measure called Abundance $(\Delta)$, proposed by Wong [10].

Abundance measure

As defined in (1), we computed $\Delta$ for a given cycle, comparing $f_{\text {input }}$, which is defined as the frequency of a pattern in the original dataset with $\bar{f}_{\text {random }}$, the 
mean of the frequencies of a pattern in $\mathrm{N}$ simulated random datasets. $\varepsilon$ is a pseudocount (Laplace smoothing) to prevent the ratio from exploding when frequencies are small. $\Delta$ can take values between -1 , underrepresented and +1 , overrepresented, being 0 the value for a pattern with the same representation in the original data than in the random datasets.

$$
\Delta=\frac{f_{\text {input }}-\bar{f}_{\text {random }}}{f_{\text {input }}+\bar{f}_{\text {random }}+\varepsilon}
$$

\section{Dataset simulation test}

In order to keep the same distribution of clusters as the original dataset, we randomized the edges between the clusters (the rearrangements). The randomization of the edges was performed using an adaptation of the switching method presented by Wong [10] to the graph abstraction previously described above. This method consists of repeatedly selecting two random edges A-B and C-D and exchanging the ends to form two new edges, e.g, A-D and B-C. The resulting graph keeps the same vertices and edges count. This method has a drawback: we cannot be certain when the graph is adequately randomized, but numerical studies have shown that enough random switching samples (100 x E) are adequate to achieve a randomized set, where $\mathrm{E}$ is the total number of edges across all samples [14]. Therefore, we generated 100 simulated datasets as follows: we removed the original edges of every sample and randomly assigned the same amount of edges to each sample every time.

\section{Results}

\section{Clusters of SVs from complex patterns}

The purpose of the clustering process is to join the rearrangements that belong to the same mutation event. Therefore, in order to select the optimal bandwidths and carry out the 2-step KDE clustering, we ran several experiments with different bandwidth values, observing that the resolution of a 1-step KDE clustering is limited by the size of the chromosomes; the density estimation was exactly the same using any bandwidth equal or smaller than 1000. A first inspection of the results showed low resolution, as breakpoints were being clustered despite being separated by hundreds of thousands base pairs, indicating the need to perform a second clustering to improve the resolution.

The final selected values for the method were bandwidth $1=1000$ for the first step since it ensured the maximum resolution and bandwidth $2=400$ for the second step since it showed high inter-cluster distances while still having small intra-cluster distances. As seen in Fig. 5, selecting a higher bandwidth the breakpoints were clustered with a considerable increase of the intra-cluster distance while almost not increasing the inter-cluster distance. Opposite, selecting a lower bandwidth the behavior was smaller intra-cluster distance but with a significant decrease in the inter-cluster distance.

To determine whether the obtained clusters were composed by random rearrangements, we first analyzed the distribution of the breakpoints in the original dataset. After comparing the dispersion of breakpoints in the simulated datasets with the dispersion from the original dataset, we got a $p$-value of smaller than $1^{-5}$, indicating 
that the breakpoint locations were not following a random distribution in the cancer genomes. Furthermore, we compared the cluster density in the simulated data and the original dataset finding that the cluster density of the original dataset was unlikely obtained in a random simulation $\left(p\right.$-value $\left.<1^{-5}\right)$. Therefore, the clusters we obtained implementing the 2-step KDE clustering contain SVs that are likely mechanically linked and not just random occurrences.

\section{Motif Finding}

Using the graph mining technique allowed us to to convert our pattern search across all the genome of every sample in a simpler graph search. Within High Performing Computing environments that are based on Apache HBase [15], HDFS [16] and Spark [17] we are able to distribute the computational load across several machines. We used three machines with an Intel@ Xeon(R) CPU E5-2630 v4@2.20GHz processor, $128 \mathrm{MB}$ of RAM, and 20 cores each. Using these technologies, the search across 2392 samples was done in less than a day. The use of High Performing Computing methods becomes crucial for the analysis of simulated datasets, where we must repeat the methodology for 100 simulations.

Here, we only focused on cycles limited to a size of 6 . The cycle with a size of 3 , named triangle, was the pattern more recurrent across the different cancer samples. Its confidence was almost twice the confidence of the next simplest cycle, composed of only 1 edge more (Table 1 ).

\begin{tabular}{lccc} 
Cycle size & Confidence & Average & Frequency \\
\hline 3 & 814 & 4.68 & 3817 \\
4 & 417 & 6.75 & 2817 \\
5 & 260 & 4.04 & 1051 \\
6 & 188 & 44.43 & 8354
\end{tabular}

Table 1 Statistical values for the evaluated cycles. The values obtained are defined as follows.

Confidence, which provides the number of samples that have at least one cycle occurrence. Average which refers to the average of the number of cycles happening in the samples. And finally, frequency, the sum of all the occurrences of the cycle across the whole dataset.

The challenge in the identification of complex patterns is to discern between the distributions of rearrangements that are the sum of random unrelated occurrences from those that are mechanically associated. We measured the significance of the patterns by calculating the Abundance $(\Delta)$. All the cycles evaluated in this study were overrepresented as shown in Fig. 6 (all cycles got positive values of Abundance.) However, as the number of rearrangements of the cycle increased, the Abundance decreased, being the triangle, the most overrepresented pattern.

\section{Pattern significance across cancer types}

Analyzing the behavior of the cycles in each cancer type, the abundances differed between tumor types (see Fig. S1). The triangle pattern again predominated over the majority of cancers, with the exceptions of Bone-Osteosarc, Kidney-ChRCC, Lymph-CLL, and Uterus-AdenoCA. Furthermore, there are tumor types that were more similar in terms of abundances of particular cycles. For example, Bladder-TCC, Bone-Osteosarc, Breast-AdenoCA, Breast-LobularCA, ColoRect-AdenoCA, Eso-AdenoCA, Head-SCC, Kidney-ChRCC, Lung-AdenoCA, Lung-SCC, Ovary-AdenoCA, Panc-AdenoCA, Prost-AdenoCA, SoftTissue-Leimyo, 
Stomach-AdenoCA, Uterus-AdenoCA had high Abundance for most of the cycles. In contrast, Breast-DCIS, Cervix-AdenoCA, Myeloid-AML, Myeloid-MPN had Abundance $=0$ for every cycle or almost every cycle. This group was clearly composed of cancer types without enough samples or complexity. The rest of the cancer types lied somewhere in the middle, having Abundance values not as high as the first group but not having all of them to 0 either: Biliary-AdenoCA, Bone-Benighm, BoneEpith, CNS-GBM, CNS-Medullo, CNS-Oligo, CNS-PiloAstro, Cervix-SCC, KidneyRCC, Liver-HCC, Lymph-BNHL, Lymph-CLL, Panc-Endocrine, Skin-Melanoma, SoftTissue-Liposarc, Thy-AdenoCA.

\section{Characterization of Triangle types}

We further characterized the triangle pattern since it was the most overrepresented and recurrent across all the samples. Known patterns of structural variants that could coincide with these triangles have been described based on the orientation of chromosomal segments at the breakpoints and their associated copy-number alterations. Using these criteria, we subclassified the triangle patterns into four different categories: i) Chromoplexy described by Baca et al. [3] where usually there is not DNA gain and even, there could be a minimal loss (balanced rearrangements); ii) Cycles of templated insertions, characterized by copy number gains and inverted rearrangements [5]; iii) Non-canonical chromothripsis, a pattern that was recently described [18], which can involve different chromosomes with frequently inverted rearrangements with oscillating copy-number alterations; iv) The fourth pattern, that we here have called Chromotrikona (from the Greek chromo for chromosome and from the Sanskrit trikona for triangle), do not correspond to any other pattern previously described and is characterized by the presence of frequent inverted rearrangements with no significant gains or losses of DNA.

Once we set the four classes of triangles, their abundances were estimated (see Fig. S2). Since we already knew that triangles were overrepresented, we expected to have a high abundance in all types. However, we noticed that Chromoplexy and Chromotrikona patterns were the most overrepresented types. These abundance similarities may be generated due to an overlapping of triangles of both types, having one or more clusters in common. Since we knew that clusters could have more than one breakpoint, they could be linked to different clusters, forming different triangles and therefore, different triangle types. We calculated the number of clusters that had in common every pair of triangles (see Fig. S3). As expected, Chromoplexy patterns had more common clusters with Chromotrikona patterns. Furthermore, this behavior was also maintained for Cycles of templated insertions and Non-canonical chromothripsis. These results suggest that these patterns could share some underlying properties as they are found in the same genomic regions.

We also performed an analysis of how these triangle types were distributed among the different cancer types. We excluded cancer types having less than 10 samples with triangles to avoid possible bias due to the low number of samples. The presence of the triangle types were heterogeneous across cancer types (Fig. 7). For instance, Chromoplexy was more common than the other triangle types in Lymph-BNHL, Uterus-AdenoCA, Panc-AdenoCA, Head-SCC, Ovary-AdenoCA, Prost-AdenoCA, and Breast-AdenoCA, while Cycles of Templated Insertions was predominant in 
Bone-Osteosarc or Skin-Melanoma. Chromotrikona predominated only in KidneyRCC and was the less represented pattern in Bone-Osteosarc, Liver-HCC, HeadSCC, Skin-Melanoma and SoftTissue-Liposarc.

\section{Discussion}

The identification and classification of complex patterns in cancer genomes are not well explored. The complexity of the data and the lack of certainty about the relevant cases claims new strategies that allow us to get insights into their underlying role in tumorigenesis.

Here we have proposed a statistical framework to fulfill this gap. First, we used a KDE-based clustering method identifying adjacent SVs that are not independent events but belonged to the same single event. The KDE clustering has been proven to be fast and simple and very suitable for distribution based-clustering tasks without setting a priori number of clusters [19, 20]. Facing the lack of reference complex patterns of SVs to compare with, we presented a statistical approximation to prove that the clusters of SVs were not by chance, indicating that they must be related to each other $[21,22]$.

For the detection of motifs to identify the complex chromosomal rearrangements, we adapted a graph mining strategy with a measure of significance for each found pattern [23]. Similar motif finding algorithms based on randomizations have been already proved successfully such as FANMODE [24], MODA [25], and NetMode[26]. All these studies agree that the need to apply the methods to both the original and simulated datasets translates into a high computational burden. We used parallelization and HPC tools to decrease the computational cost of the method [27], as well as narrow down the search to patterns of size 6 . The selected measure for analyzing the significance of the motifs, the Abundance, is directly related to the $z$-score of the pattern but normalized, allowing us to compare among different patterns [28].

Taken together, we here present the development and application of a new unbiased methodology for the classification of complex SV patterns in tumor genomes. Applying this method to more than 150 thousand SVs from the PCAWG cohort we could identify existing known patterns, as well as a new pattern (Chromotrikona) composed of three SVs that involves balanced inversions between distinct DNA regions in 2 or 3 chromosomes. This represents a significant step forward towards the understanding of the role of complex structural rearrangements in cancer.

\section{Conclusions}

In this study, we presented the development of a new statistical strategy for the classification of complex rearrangements in cancer, which is key to understanding the role and the impact of structural variation in the origin and evolution of tumors. Considering the current expansion of AI approaches for the analysis of complex biological data, this study highlights the necessity to establish robust, unbiased, and accurate statistical frames that are the foundation of more complex machine learning algorithms.

The new strategy proposed in this study fulfilled this end, being composed of an unbiased clustering solution based only on the data distribution, a robust motif finding algorithm that can be easily parallelizable to decrease the computational 
cost of such an extensive search and a final statistical measure that accurately ranks the obtained patterns in terms of significance.

The results showed the identification of different known patterns in cancer samples as well as a new pattern not previously described. This recurrent pattern, called Chromotrikona, is defined by inverted rearrangements where there are no significant gains or losses of DNA. The development of methods for studying complex patterns of SVs allows us to have insights into new patterns but also understand the genesis of chromosomal rearrangements without limited resolutions. Such genomic rearrangements are the result of subverted biological processes by which they contribute to cancer development.

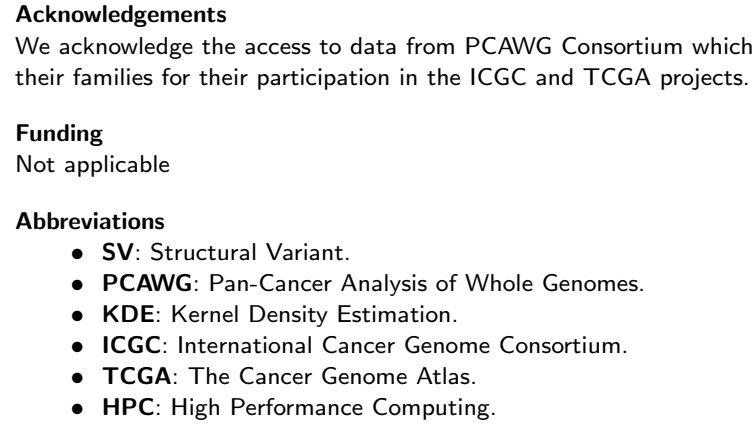

Availability of data and materials

All the data analyzed during the current study are available in the data repositories from ICGC data portal.

Ethics approval and consent to participate

Not applicable.

Competing interests

The authors declare that they have no competing interests.

Consent for publication

Not applicable.

Authors' contribution

GG and JL designed the methodology. GG developed the methods and performed the main statistical analysis. LD characterized the triangle patterns. GG and LD conceived the work and wrote the paper. All authors read and approved the final manuscript.

Author details

${ }^{1}$ Barcelona Supercomputing Center (BSC), Department of Computer Science, Barcelona, Spain. ${ }^{2}$ Universitat Politècnica de Catalunya (UPC), Barcelona, Spain. ${ }^{3}$ Barcelona Supercomputing Center (BSC), Department of Life Sciences, Barcelona, Spain. ${ }^{4}$ Institució Catalana de Recerca i Estudis Avançats (ICREA), Barcelona, Spain.

References

1. Boveri, T.: Concerning the origin of malignant tumours by theodor boveri. translated and annotated by henry harris. Journal of cell science 121(Supplement 1), 1-84 (2008)

2. Stephens, P.J., Greenman, C.D., Fu, B., Yang, F., Bignell, G.R., Mudie, L.J., Pleasance, E.D., Lau, K.W., Beare, D., Stebbings, L.A., et al.: Massive genomic rearrangement acquired in a single catastrophic event during cancer development. cell 144(1), 27-40 (2011)

3. Baca, S.C., Prandi, D., Lawrence, M.S., Mosquera, J.M., Romanel, A., Drier, Y., Park, K., Kitabayashi, N., MacDonald, T.Y., Ghandi, M., et al.: Punctuated evolution of prostate cancer genomes. Cell 153(3), 666-677 (2013)

4. The, I., of Whole, T.P.-C.A., Consortium, G., et al.: Pan-cancer analysis of whole genomes. Nature 578(7793), $82(2020)$

5. Li, Y., Roberts, N.D., Wala, J.A., Shapira, O., Schumacher, S.E., Kumar, K., Khurana, E., Waszak, S., Korbel, J.O., Haber, J.E., et al.: Patterns of somatic structural variation in human cancer genomes. Nature 578(7793), 112-121 (2020)

6. Sheather, S.J.: Density estimation. Statistical science, 588-597 (2004)

7. Kim, J., Scott, C.D.: Robust kernel density estimation. The Journal of Machine Learning Research 13(1), 2529-2565 (2012)

8. Dowd, K., Severance, C.: High performance computing (2010)

9. Hager, G., Wellein, G.: Introduction to high performance computing for scientists and engineers (2010) 
10. Wong, E., Baur, B., Quader, S., Huang, C.-H.: Biological network motif detection: principles and practice. Briefings in bioinformatics 13(2), 202-215 (2011)

11. Jones, M.C., Marron, J.S., Sheather, S.J.: A brief survey of bandwidth selection for density estimation. Journal of the American statistical association 91(433), 401-407 (1996)

12. Chiu, S.-T.: Bandwidth selection for kernel density estimation. The Annals of Statistics, 1883-1905 (1991)

13. Kuramochi, M., Karypis, G.: Finding frequent patterns in a large sparse graph. Data mining and knowledge discovery 11(3), 243-271 (2005)

14. Milo, R., Kashtan, N., Itzkovitz, S., E. J. Newman, M., Alon, U.: On the uniform generation of random graphs with prescribed degree sequences. Tech rep 21 (2004)

15. Team, A.H.: Apache hbase reference guide. Apache, version 2(0) (2016)

16. Borthakur, D., et al.: Hdfs architecture guide. Hadoop Apache Project 53(1-13), 2 (2008)

17. Spark, A.: Apache spark. Retrieved January 17, 2018 (2018)

18. Cortés-Ciriano, I., Lee, J.J.-K., Xi, R., Jain, D., Jung, Y.L., Yang, L., Gordenin, D., Klimczak, L.J., Zhang, C.-Z., Pellman, D.S., et al.: Comprehensive analysis of chromothripsis in 2,658 human cancers using whole-genome sequencing. Nature genetics 52(3), 331-341 (2020)

19. Matioli, L., Santos, S., Kleina, M., Leite, E.: A new algorithm for clustering based on kernel density estimation Journal of Applied Statistics 45(2), 347-366 (2018)

20. Zhou, Z., Si, G., Zhang, Y., Zheng, K.: Robust clustering by identifying the veins of clusters based on kernel density estimation. Knowledge-Based Systems 159, 309-320 (2018)

21. Odén, A., Wedel, H., et al.: Arguments for fisher's permutation test. Annals of Statistics 3(2), 518-520 (1975)

22. Ojala, M., Garriga, G.C.: Permutation tests for studying classifier performance. Journal of Machine Learning Research 11(6) (2010)

23. Wong, E., Baur, B., Quader, S., Huang, C.-H.: Biological network motif detection: principles and practice. Briefings in bioinformatics 13(2), 202-215 (2012)

24. Wernicke, S., Rasche, F.: Fanmod: a tool for fast network motif detection. Bioinformatics 22(9), 1152-1153 (2006)

25. Omidi, S., Schreiber, F., Masoudi-Nejad, A.: Moda: an efficient algorithm for network motif discovery in biological networks. Genes \& genetic systems 84(5), 385-395 (2009)

26. Li, X., Stones, D.S., Wang, H., Deng, H., Liu, X., Wang, G.: Netmode: Network motif detection without nauty. PloS one 7(12), 50093 (2012)

27. Kim, W., Diko, M., Rawson, K.: Network motif detection: Algorithms, parallel and cloud computing, and related tools. Tsinghua science and technology 18(5), 469-489 (2013)

28. Milo, R., Itzkovitz, S., Kashtan, N., Levitt, R., Shen-Orr, S., Ayzenshtat, I., Sheffer, M., Alon, U.: Superfamilies of evolved and designed networks. Science 303(5663), 1538-1542 (2004)

Figures

Fig. 1 Workflow applied to identify complex rearrangements in PCAWG genomes. Simple data pre-processing was performed before implementing the recursive clustering. Then, the graph mining method was applied to find patterns. Finally, the motif finding strategy was applied to determine the statistically significant patterns

Fig. 2 Kernel Density Estimation of breakpoint clusters from chromosome 3 setting bandwidth values of (a) 3000 and (b) 8000 . Blue dots represent the locations of the breakpoints, the blue line is the kernel density estimation and red lines the obtained cluster peaks. The inter and intra-cluster distances are shown in green and red, respectively

Fig. 3 Circular representation of human genome with cycles of different sizes

Fig. 4 Graphic representation of the subgraph mining process. We performed the search for every vertex of the sample until every possible connection of size 6 was found. Since we did not implement any control during the algorithm, every pattern was likely to be found more than one time and had to be reduced in the following step. This method allowed us to parallelize the search in several machines to reduce computational time 
Fig. 5 Total inter and intra-cluster distances for the whole dataset using the 2-step KDE clustering with different bandwidth values

Fig. 6 Abundance values for the analyzed cycles. Its value can go from -1 , underrepresented, to +1 , overrepresented. The Abundance of a single rearrangement (1 SV) is also shown as a control value. Its value is 0 since we fix the rearrangements during the simulation of the random datasets, which means that its representation is the same in every dataset

Fig. 7 Confidence intervals of the mean of the frequency for each triangle type throughout cancer types. Only cancers with more than 10 samples with triangles were showed

\section{Additional Files}

Additional file 1 (pdf)

Figures S1 showing the abundance values of the evaluated cycles for the 36 cancer types. Figure S2 showing the abundance values for different triangle categories and Figure S3 showing common clusters between every pair of triangle types. 
Figures

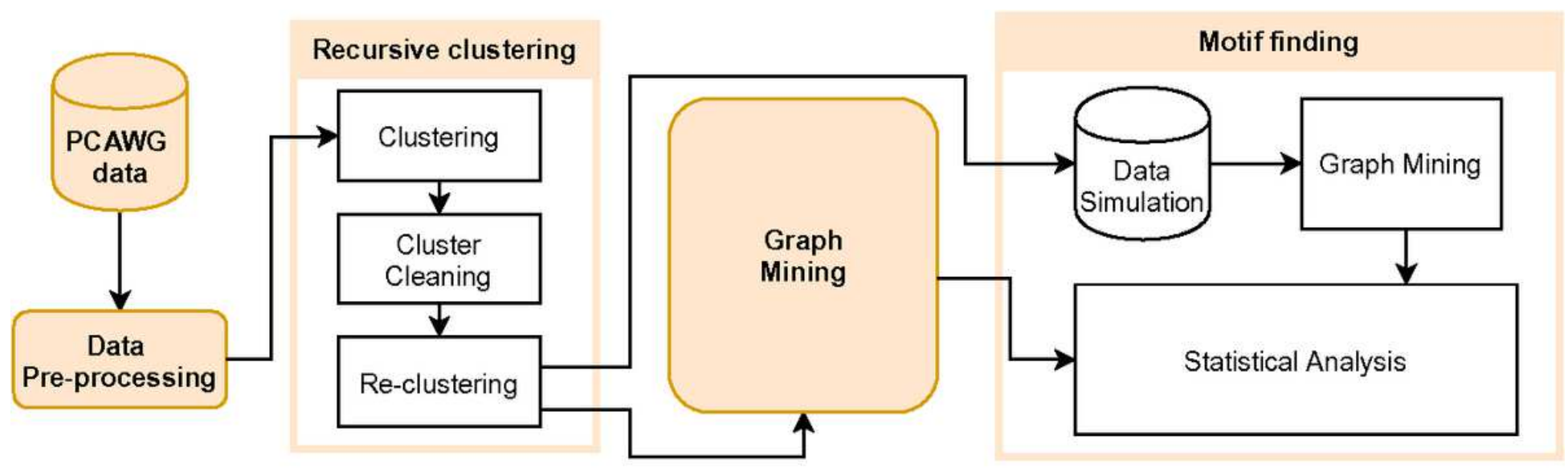

Figure 1

Workflow applied to identify complex rearrangements in PCAWG genomes. Simple data pre-processing was performed before implementing the recursive clustering. Then, the graph mining method was applied to find patterns. Finally, the motif finding strategy was applied to determine the statistically significant patterns 
(a) Bandwidth $=3.000$

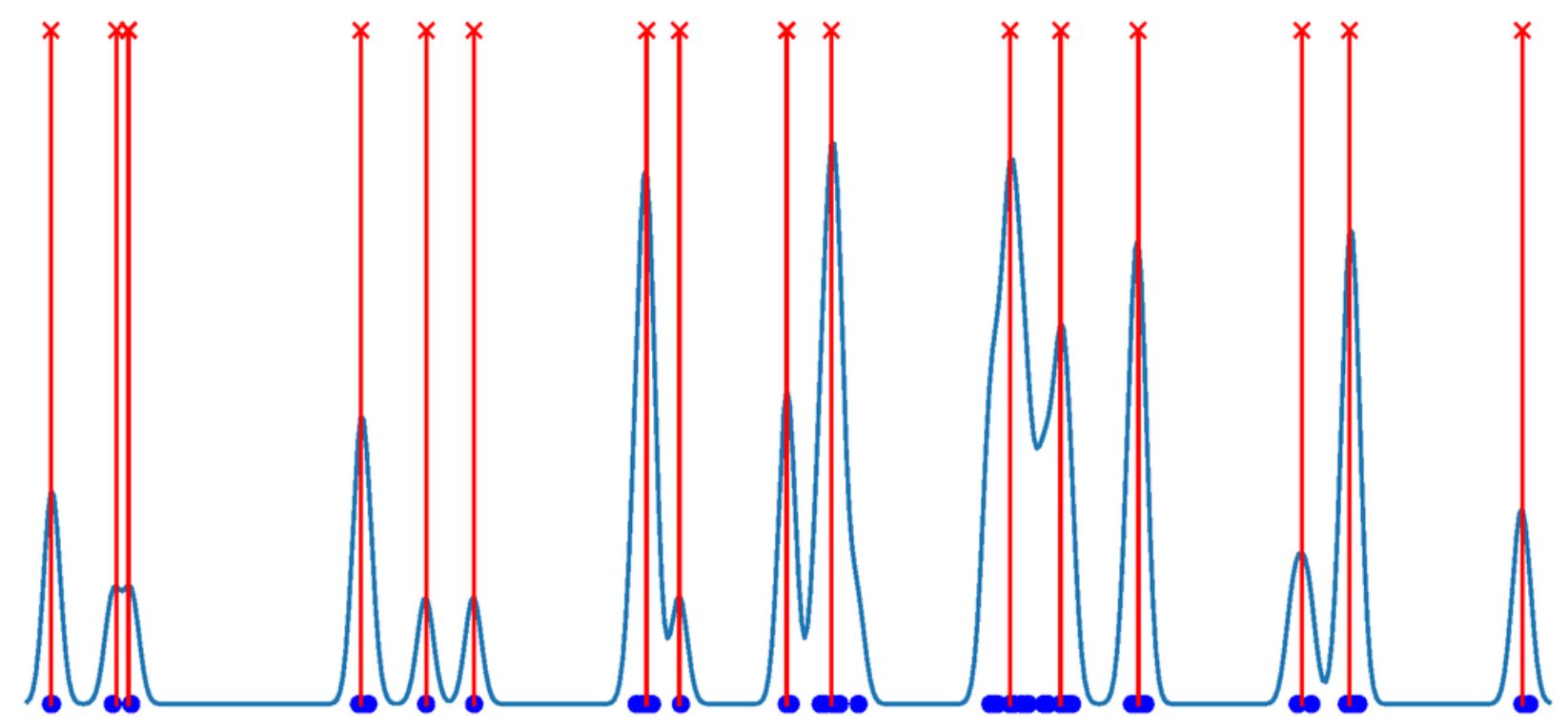

(b) Bandwidth $=8.000$

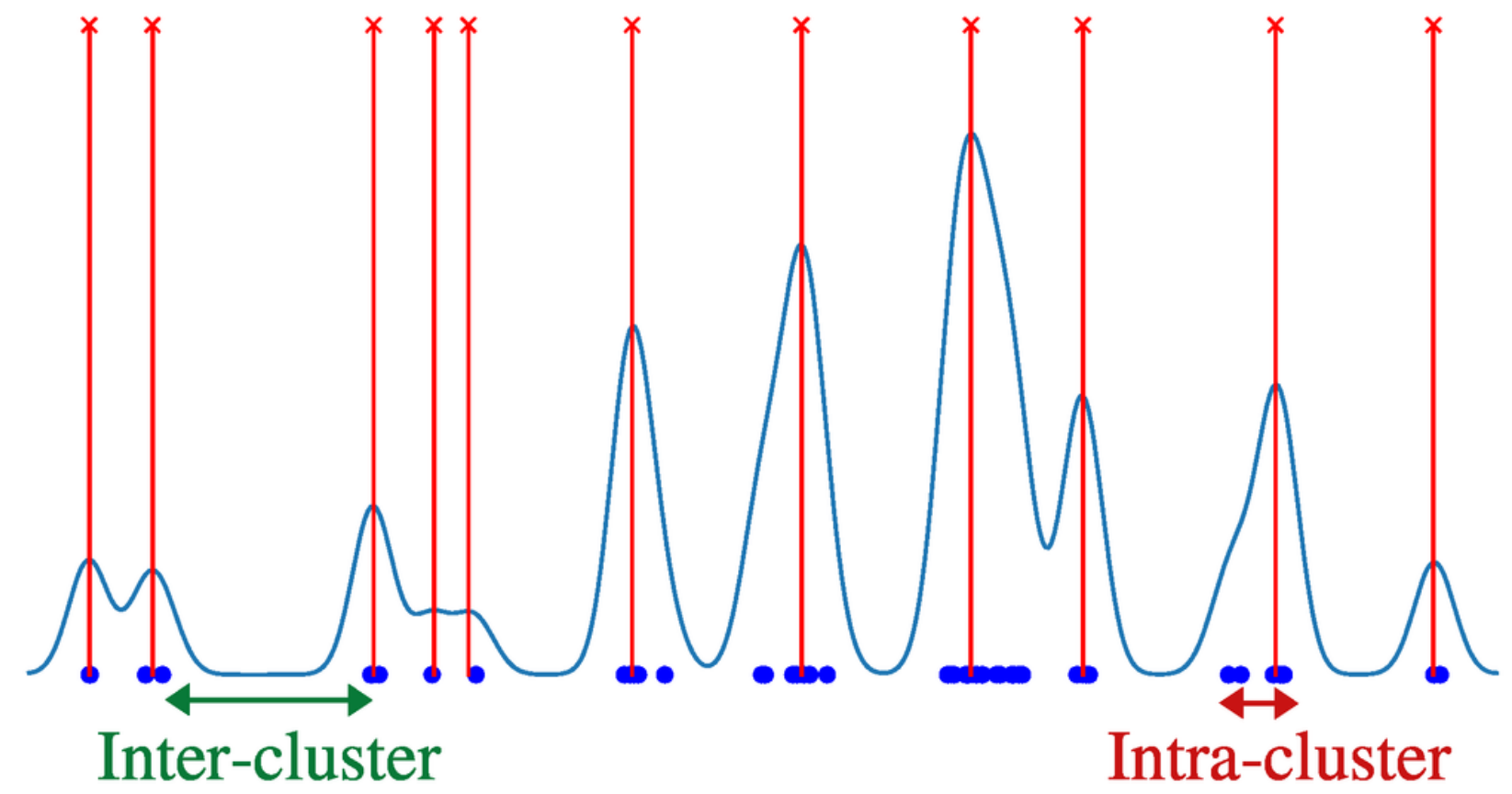

Figure 2

Kernel Density Estimation of breakpoint clusters from chromosome 3 setting bandwidth values of (a) 3000 and (b) 8000 . Blue dots represent the locations of the breakpoints, the blue line is the kernel density estimation and red lines the obtained cluster peaks. The inter and intra-cluster distances are shown in green and red, respectively 


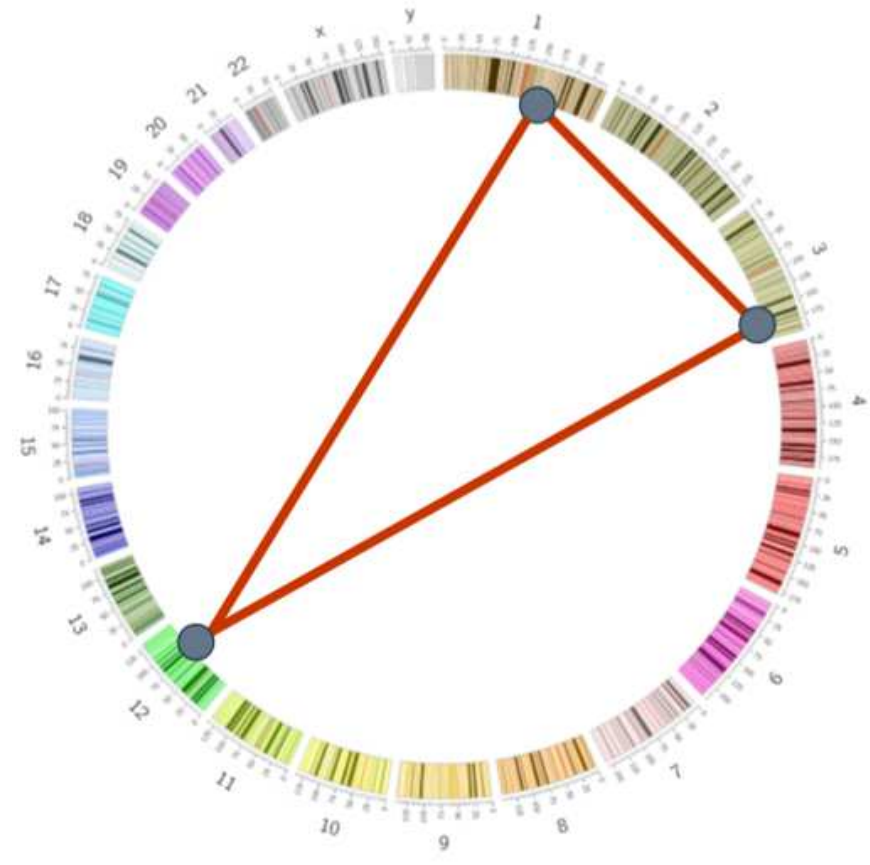

a) 3 edges

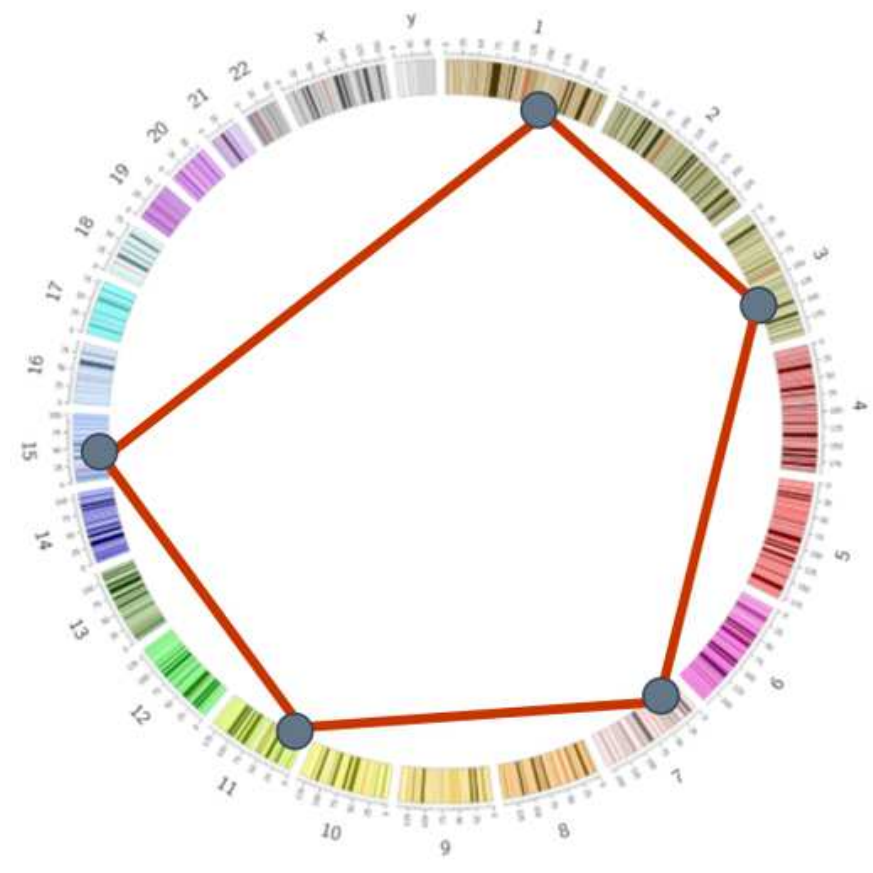

c) 5 edges

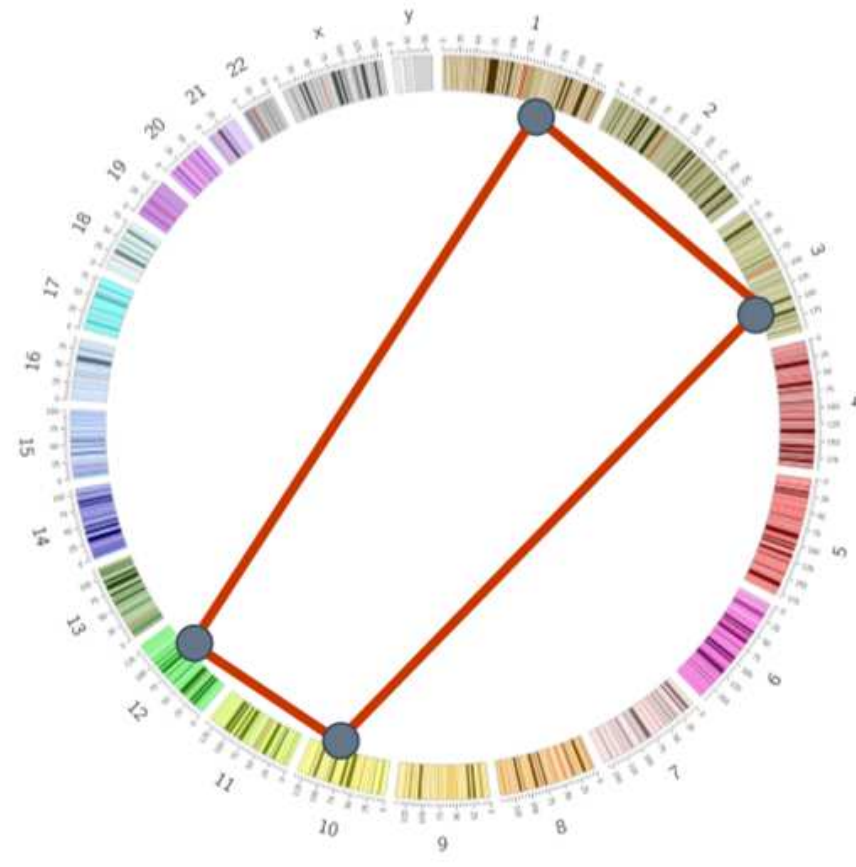

b) 4 edges

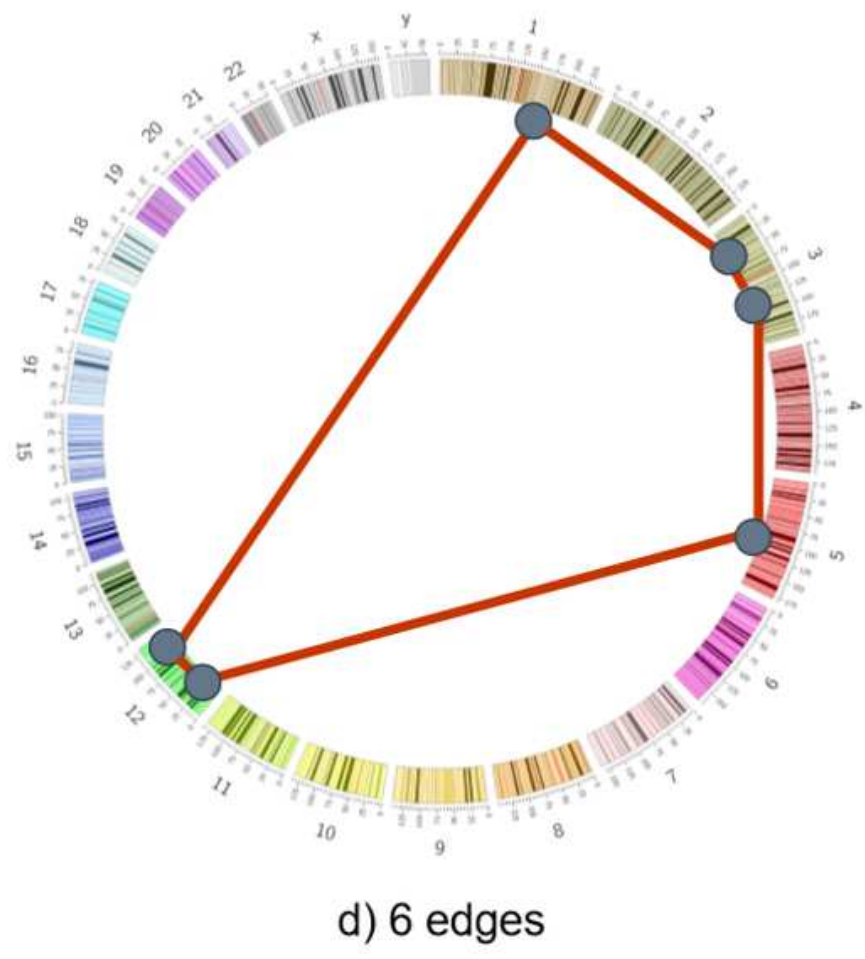

\section{Figure 3}

Circular representation of human genome with cycles of different sizes 

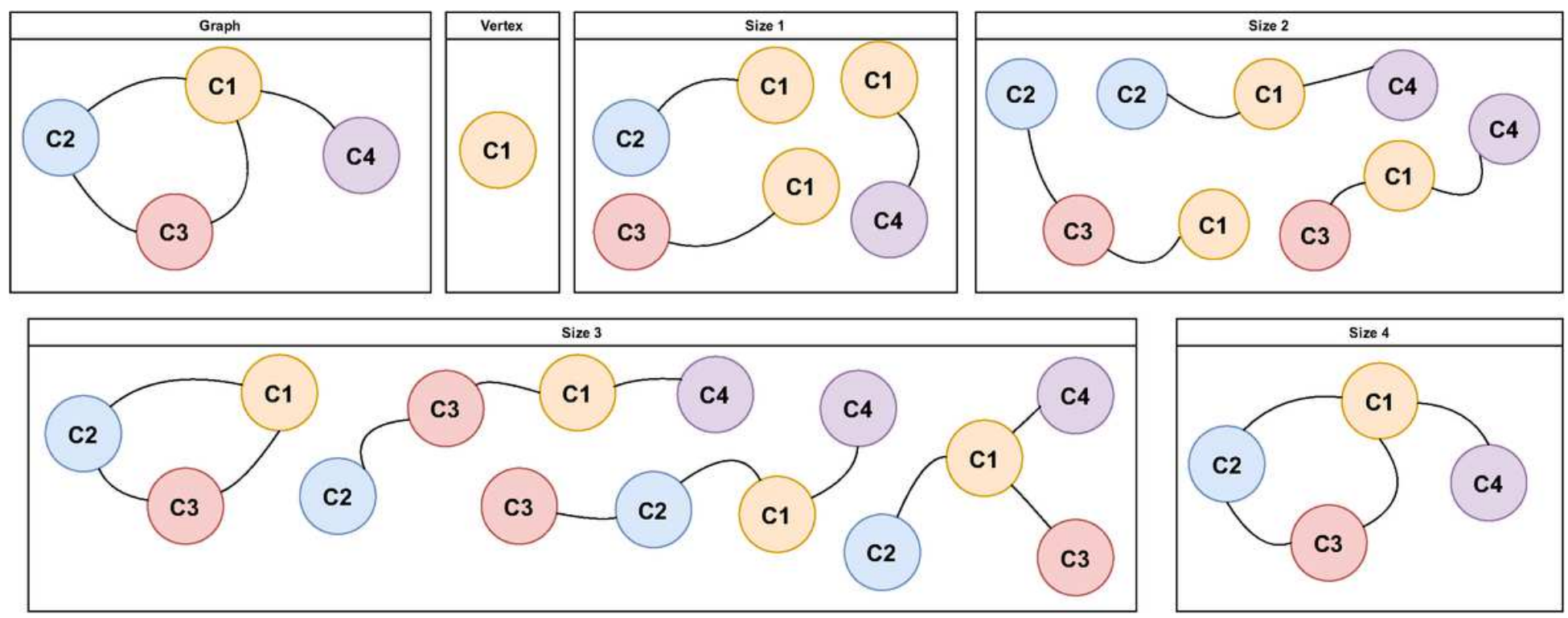

Figure 4

Graphic representation of the subgraph mining process. We performed the search for every vertex of the sample until every possible connection of size 6 was found. Since we did not implement any control during the algorithm, every pattern was likely to be found more than one time and had to be reduced in the following step. This method allowed us to parallelize the search in several machines to reduce computational time 


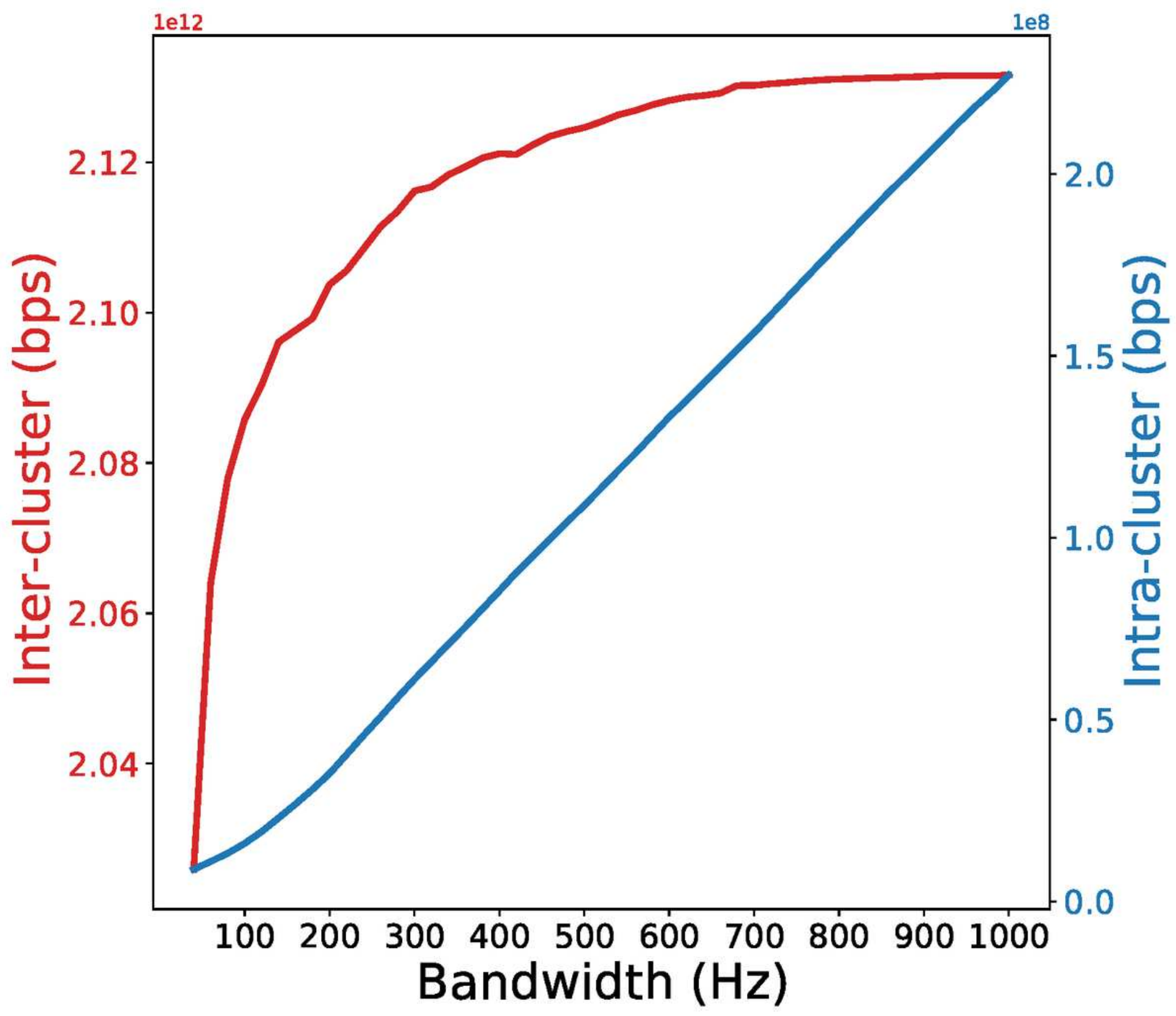

Figure 5

Total inter and intra-cluster distances for the whole dataset using the 2-step KDE clustering with different bandwidth values 


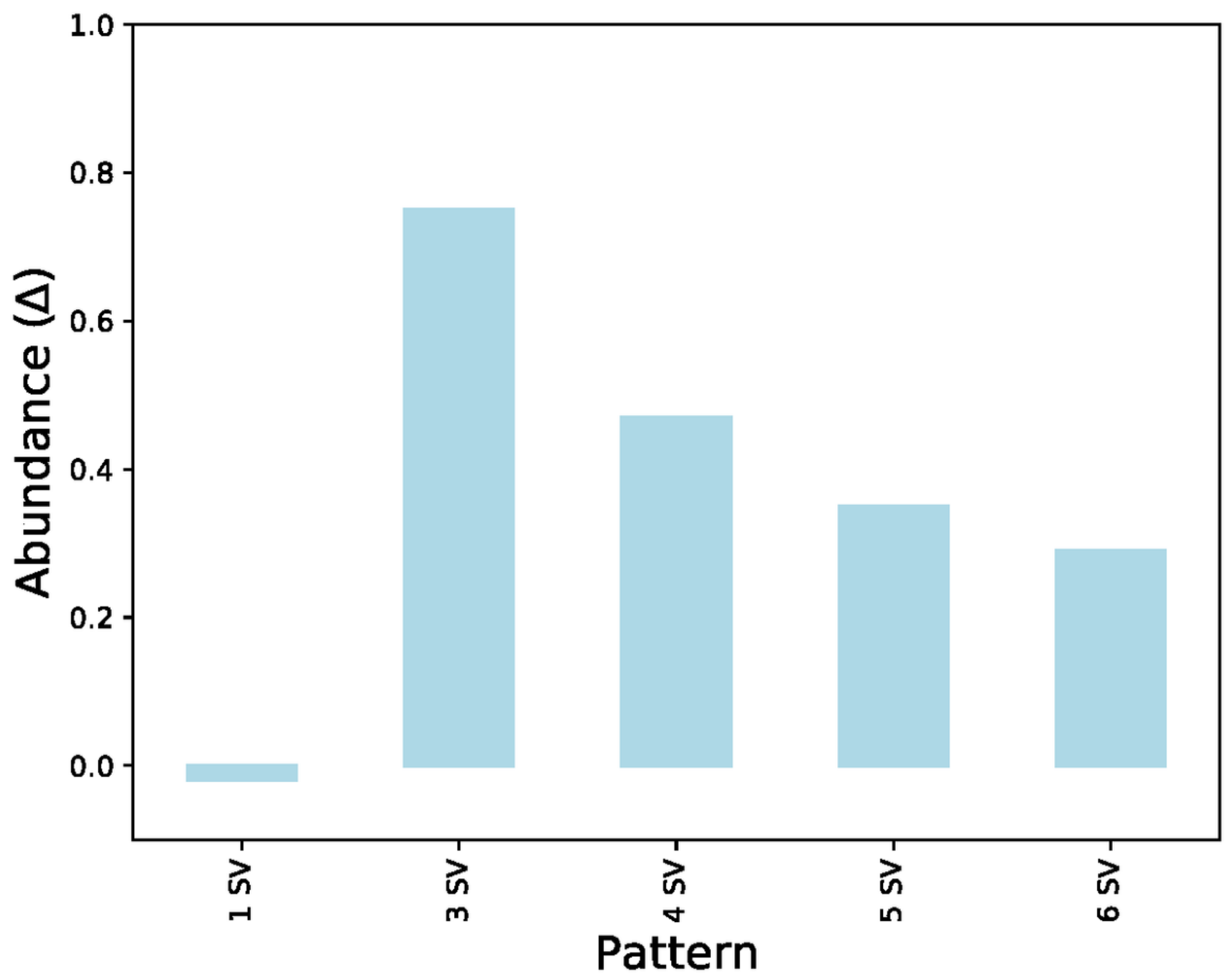

Figure 6

Abundance values for the analyzed cycles. Its value can go from -1 , underrepresented, to +1 , overrepresented. The Abundance of a single rearrangement (1 SV) is also shown as a control value. Its value is 0 since we fix the rearrangements during the simulation of the random datasets, which means that its representation is the same in every dataset 


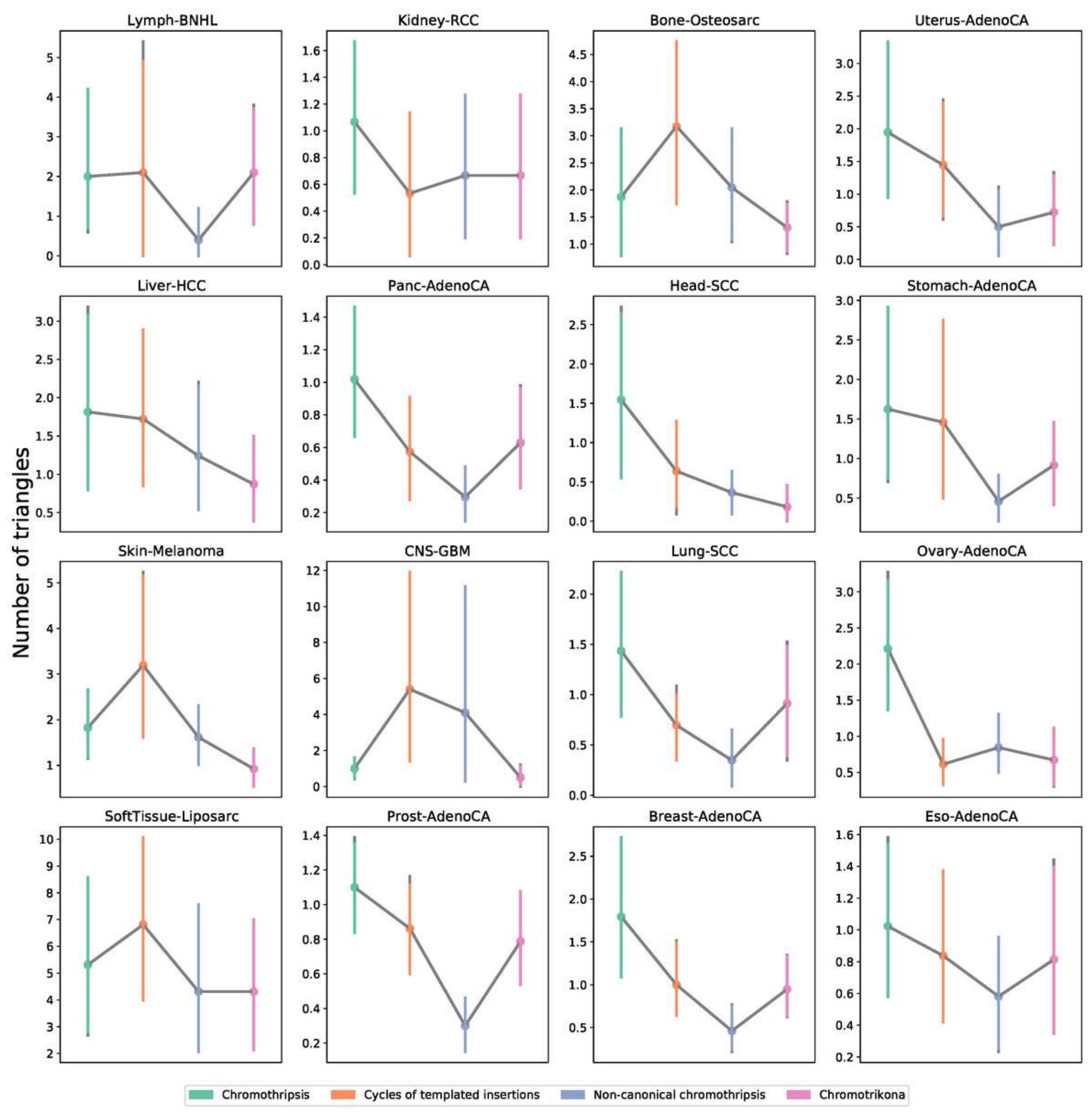

Figure 7

Confidence intervals of the mean of the frequency for each triangle type throughout cancer types. Only cancers with more than 10 samples with triangles were showed

\section{Supplementary Files}

This is a list of supplementary files associated with this preprint. Click to download. 
- AdditionalFile1.pdf 